\title{
Examining Gross Anatomy Study Strategies Across Old and New Curricula
}

\author{
Elizabeth Davis, B.A. ${ }^{1}$, Polly R. Husmann, Ph.D. ${ }^{2}$ \\ ${ }^{1}$ Indiana University School of Medicine, ${ }^{2}$ Medical Sciences Program, Indiana \\ University School of Medicine
}

Background and Hypothesis: In the fall of 2016, Indiana University School of Medicine implemented a new integrated curriculum. We hypothesized that the study strategies utilized by students for gross anatomy changed due to this redesign.

Project Methods: Students were given a voluntary survey about their study strategies, attitudes, and basic demographics near the end of their anatomy course. The students' NBME and final course scores were also linked to their responses and responses were condensed into categories. Comparisons were made between students in the upper and lower thirds of the classes and between old and new curriculum cohorts.

Results: Several differences were found between the old and new curricula: use of web-based resources increased, self-quizzing decreased, and attendance decreased at a statistically significant level $(p<0.0001, p=0.042, p<0.0001)$. Across both curricula, students who were more confident that they had studied enough going into the tests performed more poorly than those who were less confident $(p<0.0001)$. Consistently the students in the top third of the class demonstrated total amounts of study strategies that were lower than in the bottom third of the class, though these results did not reach statistical significance $(p=0.382)$.

Conclusion and Potential Impact: Student study strategies have changed between the previous and current anatomy courses in ways that may impact future student outcomes. More use of web-based resources may be of questionable quality and lead to more students feeling overwhelmed, while less self-quizzing may lead to less long-term retention of anatomy content. Metacognitive skills remain vital across curricula. 